\title{
Acupuncture treatment for patients with asthma
}

\author{
Zogopoulos $\mathrm{P}^{1,2 *}$, Sagris $\mathrm{K}^{3}$, Gkorgkolis $\mathrm{V}^{4}$, Kollias $\mathrm{S}^{2}$, Pankos $\mathrm{A}^{2}$, Vretakos $\mathrm{G}^{2}$ and Rologis $\mathrm{D}^{2}$ \\ ${ }^{1}$ Department of Medical Acupuncture, Metropolitan Hospital, Athens, Greece \\ ${ }^{2}$ Department of Neurosurgery, Metropolitan Hospital, Athens, Greece \\ ${ }^{3}$ Intensive Care Unit, Metropolitan Hospital, Athens, Greece \\ ${ }^{4}$ St. Andrews Centre for Plastic Surgery and Burns, Broomfield Hospital, Chelmsford, UK
}

\begin{abstract}
Acupuncture is a treatment modality for a wide range of disorders and diseases. Although, it has been widely practised in Asia for many centuries, western medicine has focused on its therapeutic potential only over the last few decades. Extensive investigations are currently being conducted worldwide, regarding acupuncture's indications, efficacy and mechanisms of action. We present a review of available experimental and clinical data, to date, regarding acupuncture's efficacy as an adjunctive treatment for patients with asthma.
\end{abstract}

\section{Introduction}

Bronchial asthma is a chronic airway inflammatory disease, characterized by airway hyperresponsiveness that leads to airflow obstruction or even bronchospasm, and chronic inflammation [1]. Common symptoms include coughing, wheezing, shortness of breath and chest tightness [2]. Acupuncture treatment has been proven effective for many different diseases and disorders, including airway disorders such as bronchial asthma.

\section{Materials and methods}

We searched all available publications on electronical medical database PubMed, regarding acupuncture's efficacy in treating patients with asthma. Search terms were "acupuncture" and "asthma". In total, 27 studies met the inclusion criteria (16 clinical and 11 experimental studies).

\section{Results}

\section{Experimental data}

Many experimental animal studies (mostly on rats and mice) have documented the efficacy of acupuncture treatment in alleviating asthma symptoms and have shed light on the mechanisms of action and the neurobiology behind these beneficial effects. Most commonly used experimental model is that of ovalbumin-induced bronchial asthma.

Specifically, acupuncture treatment led to a significant suppression of airway hyperresponsiveness, chronic lung inflammation and mucus secretion in animal models of asthma (after injection and inhalation of ovalbumin) [3,4]. It also inhibited airway remodeling, decreased airway resistance and increased lung dynamic compliance, since acupuncture treatment resulted in a remarkable decrease of the airway wall thickness and smooth muscle thickness [4,5]. These effects were mediated by an inhibition of $\mathrm{Ca}(\mathrm{v}) 3.1$ T-type calcium channel protein expression in airway smooth muscle cells [6].

A significant decrease in serum concentrations of certain proinflammatory cytokines, such as interleukin (IL)-1 $\beta$, IL-33 and tumor necrosis factor $-\alpha$ (TNF- $\alpha$ ) compared to untreated asthmatic mice (control group) was also observed after acupuncture treatment [4]. Moreover, acupuncture reduced the CD4 $+\mathrm{IL}-17 \mathrm{~A}+$ cell proportion and counts and increased CD4+Foxp3+ cell counts in bronchoalveolar lavage fluid $[3,4]$. Acupuncture also reduced ovalbumin-specific $\operatorname{IgE}$ levels, as well as Th17 cytokine levels (such as IL-17A, IL-17F and IL22) in the serum, compared to control group [3].

Hematoxylin and eosin staining of bronchoalveolar lavage fluid after acupuncture treatment has revealed significantly reduced inflammatory infiltration and aggregation of a variety of inflammatory cells (eosinophilic granulocytes, neutrophils and lymphocytes) around airways, compared to control group [3,7-9]. These beneficial effects of acupuncture on asthma treatment may be mediated by suppression of acetylcholine signalling both during its synthesis, as well as its release, since acupuncture treatment reduced acetylcholine synthetase pulmonary expression and restored pulmonary expression of muscarinic receptors (mAchRs) M1 \& M2 (especially in the bronchiolar epithelium), that play a pivotal role in acetylcholine release inhibition [9]. Furthermore, acupuncture significantly reduced expression of protein kinase $\mathrm{B}(\mathrm{PKB} / \mathrm{AKT})$ in lung tissues and consequently reduced inflammatory reaction and airway remodelling [8].

Acupuncture treatment in experimental rat asthma models significantly inhibited transforming growth factor $\beta 1$ (TGF- $\beta 1$ ) expression in the lung tissues (both in bronchoalveolar lavage fluid and serum) compared to the control group $[5,10]$. Moreover, TGF- $\beta 1$ expression levels were even lower than those of the medication group (aminophylline administration), thus, suggesting that this effect could play a pivotal role in airway remodeling improvement [5]. TGF- $\beta 1$ is a polypeptide that belongs to the family of cytokines and performs many cellular functions (such as control of cell proliferation, differentiation

${ }^{\star}$ Correspondence to: Zogopoulos Panagiotis, Department of Medical Acupuncture, Metropolitan Hospital, Athens, Greece, E-mail: p.zogopoulos@yahoo.com

Received: September 06, 2018; Accepted: September 20, 2018; Published: September 22, 2018 
and apoptosis), especially regarding the immune system [11]. In another ovalbumin-induced murine asthma model, acupuncture treatment enhanced hypothalamic-pituitary-adrenal (HPA) axis activity and thus, increased adrenocorticotropic hormone and cortisol secretion in the plasma [12]. These effects on TGF- $\beta 1$ expression and HPA-axis activity could explain, at least in part, the anti-inflammatory properties of acupuncture and particularly the attenuation of airway inflammation in asthma patients [12].

Protein electrophoresis and mass spectrometry in experimental animal models of asthma have shown that acupuncture can regulate the inflammation signaling pathway at several different key points by regulating specific proteins [13]. In particular, it can downregulate proinflammatory proteins [such as S100 calcium-binding protein A8 (S100A8), receptor for advanced glycation endproducts (RAGE) and S100 calcium-binding protein A11 (S100A11)] and upregulate anti-inflammatory proteins [such as Clara cell $10 \mathrm{kD}$ protein (CC10), Annexin-A5 (ANXA5) and soluble RAGE (sRAGE)] [13]. Furthermore, acupuncture can significantly increase Fas mRNA expression (Fas protein is a member of the TNF-receptor superfamily and is implicated in the regulation of programmed cell death), while Bcl-2 mRNA expression is significantly reduced (Bcl-2 is an integral outer mitochondrial membrane protein that blocks the apoptotic death of some cells such as lymphocytes) [7].

Clinical data: Several randomized, controlled, clinical trials in patients with asthma have proven acupuncture's clinical efficacy in terms of symptoms remission, as well as respiratory functional status. Patients with allergic asthma who received acupuncture treatment in combination with their standard medication exhibited significantly improved disease-specific and health-related quality of life (as assessed with the asthma quality of life questionnaire and Short-Form-36 questionnaire), compared to patients who received standard medication alone [14].

Randomized, controlled clinical trials in children with asthma showed that acupuncture treatment resulted in a significant reduction of subjective asthma symptoms and of inhaled steroids and $\beta 2$ agonists use, compared to the control group, for as long as acupuncture treatment was administered, but these beneficial effects were not sustained after acupuncture's discontinuation [15].

In a randomized, controlled, clinical trial in patients with mild to moderate asthma, acupuncture treatment led to a decrease in eosinophils and neutrophils and an increase in macrophages [16]. Patients experienced less coughing, wheezing, dyspnea and nocturnal awakening episodes and used significantly less rescue medication (short- and long-acting $\beta-2$ agonists and inhaled corticosteroids) [16]. Finally, patients exhibited significant improvement in peak expiratory flow, peak expiratory flow variability, functional capacity and general health status $[16,17]$.

A meta-analysis on randomized controlled clinical trials of acupuncture for asthma patients found that acupuncture can significantly improve peak expiratory flow (PEFR), forced vital capacity (FVC) and the Tiffeneau-Pinelli index (FEV1/FVC), although FEV1 was not found to be affected in a statistically significant manner [18]. However, there have been reports from randomized, controlled clinical trials that FEV1 can also be significantly improved with acupuncture, up to the same extent or even superior to inhaled bronchodilation $[19,20]$.

Acupuncture treatment in patients with bronchial asthma was found to be equally effective to inhaled salbutamol in terms of acute symptoms' control and could achieve immediate relief within 30 minutes of needle insertion [21]. It also led to a significant decrease of $\operatorname{IgA}$ concentration in the saliva and nasal secretions, as well as a significant decrease of total serum IgE, IL-2R+ T lymphocytes and eosinophils counts in the peripheral blood [22]. On the other hand, a significant increase of $\mathrm{CD} 3+, \mathrm{CD} 4+$, and $\mathrm{CD} 8+\mathrm{T}$ lymphocytes, as well as an increase of IL- 8 and a significant decrease of IL- 6 and IL10 concentrations in the peripheral blood were observed, suggesting that acupuncture can have a regulatory effect on cellular and mucosal immunity in asthma patients [22,23].

\section{Discussion}

Bronchial asthma is a serious health problem with nearly 5\%-10\% of the global population suffering from it [24]. Particularly, incidence of asthma and asthmatic bronchitis among children is estimated to be even higher (between 10\% and 20\%) [15]. Despite numerous available medications for bronchial asthma (such as inhaled corticosteroids, long acting beta agonists and antileukotriene agents), there is no definitive treatment and symptoms' control is usually only temporary or even not feasible. Therefore, new therapeutic regimens that combine both high efficacy and minimum side-effects are still necessary and acupuncture treatment can play that role.

Acupuncture treatment for allergic bronchial asthma in addition to standard care is associated with additional costs but also with greater efficacy regarding symptoms' control and, thus, improvement of quality of life as has been depicted through Asthma Quality of Life Questionnaire (AQLQ) score improvement [25,26].

There is a hypothesis that acupuncture may inhibit re-uptake of neurotrophic factors (such as brain-derived neurotrophic factor, nerve growth factor and leukemia inhibitory factor) that are synthesized in bronchial epithelial and immune cells and consequently inhibit synthesis and release of substance P in dorsal root ganglia [27]. Substance P is known to cause contraction of airway smooth muscle cells, increase of mucous secretion, capillary extravasation and release of inflammatory mediators, which all contribute to airway hyper-reactivity [27]. Thus, through the inhibition of this cascade acupuncture can exhibit its beneficial effects in asthma patients.

There have been reports in the literature that acupuncture failed to improve clinical status and functional respiratory indexes of asthma patients [28-31]. A general big concern regarding acupuncture trials is the lack of a rigorous study design, patient selection bias and poor study quality and reporting. As the interest for acupuncture is constantly increasing internationally and more and more patients seek alternative treatments for their condition, strict methodological criteria should be adopted for all trials on acupuncture and furthermore, acupuncture practice should only be restricted to experienced and specially trained physicians who can guarantee both safety and effectiveness of this treatment modality.

\section{Conclusion}

Acupuncture can effectively protect lung function and prevent airway inflammation, thereby playing an important role as an adjunctive treatment modality for asthma patients. Furthermore, it is a cost-effective treatment with no serious adverse effects, when practised by an experienced and certified acupuncturist.

\section{References}

1. National Heart Lung and Blood Institute (2007) National asthma education and prevention program. "expert panel report 3: Guidelines for the diagnosis and management of asthma 1: 11-12. 
2. British Thoracic Society (2012) British Guidelines on the Management of Asthma 4.

3. Wei Y, Dong M, Zhang H, Lv Y, Liu J, et al. (2015) Acupuncture Attenuated Inflammation and Inhibited Th17 and Treg Activity in Experimental Asthma. Evid Based Compement Alternat Med 2: 340126.

4. Dong M, Ma C, Wang WQ, Chen J, Wei Y, et al. (2018) Regulation of the IL-33/ST2 pathway contributes to the anti-inflammatory effect of acupuncture in the ovalbumininduced murine asthma model. Acupunt Med. [Epub ahead of print]

5. Li HZ, Qin XY, Shao SJ, Hua JS (2014) Effect of acupuncture intervention on airway remodeling and tranforming growth factor-betal expression in asthma rats. Zhen $C$ Yan Jiu 39: 278-281.

6. Wang Y, Sun J, Jin R, Liang Y, Liu YY, et al. (2012) Influence of acupuncture on expression of T-type calcium channel protein in airway smooth muscle cell in airway remodeling rats with asthma. Zhongguo Zhen Jiu 32: 534-540.

7. Wu ZL, Li CR, Liu ZL, Zhang QR (2012) Effects of acupuncture at "Zusanli” (ST 36) on eosinophil apoptosis and related gene expression in rats with asthma. Zhongguo Zhen Jiu 32: 721-725.

8. Yang J, Zhao Y, Li S, Han J, Yu Y, et al.(2017) Regulation of acupuncture on expression of AKT protein in lung tissues of asthma rats. Zhongguo Zhen Jiu 37: 406-410.

9. Liu YL, Zhang LD, Ma TM, Song ST, Liu HT, et al. (2018) Feishu Acupuncture Inhibits Acetylcholine Synthesis and Restores Muscarinic Acetylcholine Receptor M2 Expression in the Lung When Treating Allergic Asthma. Inflammation. [Epub ahead of print]

10. Han J, Li S, Zhao Y, Yang J, Yu Y, et al. (2017) Acupuncture for TGF- $\beta 1$ expression in lung tissues of asthma rats. Zhongguo Zhen Jiu 37: 741-746.

11. Letterio JJ, Roberts $\mathrm{AB}(1998)$ Regulation of immune responses by TGF-beta. Annu. Rev. Immunol16: 137-161.

12. Wei Y, Dong M, Zhong L, Liu J, Luo Q, et al. (2017) Regulation of hypothalamicpituitary-adrenal axis activity and immunologic function contributed to the antiinflammatory effect of acupuncture in the OVA-induced murine asthma model. Neurosci Lett 636: 177-183.

13. Xu YD, Cui JM, Wang Y, Yin LM, Gao CK, et al. (2012) Proteomic analysis reveals the deregulation of inflammation-related proteins in acupuncure-treated rats with asthma onset. Evid Based Complement Alternat Med 4: 850512.

14. Brinkhaus B, Roll S, Jena S, Icke K, Adam D, et al. (2017) Acupuncture in Patients with Allergic Asthma: A Randomized Pragmatic Trial. J Altern Complement Med 23: 268-277.

15. Karlson G, Bennicke P (2013) Acupuncture in asthmatic children: a prospective, randomized, controlled clinical trial of efficacy. Altern Ther Health Med 19: 13-19.

16. Pai HJ, Azevedo RS, Braga AL, Martins LC, Saraiva-Romanholo BM, et al. (2015) A randomized, controlled, crossover study in patients with mild and moderate asthma undergoing treatment with traditional Chinese acupuncture. Clinics (Sao Paulo) 70: 663-669.
17. Liu CF, Chien LW (2015) Efficacy of acupuncture in children with asthma: a systematic review. Ital J Pediatr 41: 48.

18. Yu L, Zhang Y, Chen C, Cui HF, Yan XK (2010) Meta-analysis on randomized controlled clinical trials of acupuncture for asthma. Zhongguo Zhen Jiu 30: 787-792.

19. Chu KA, Wu YC, Ting YM, Wang HC, Lu JY (2007) Acupuncture therapy results in immediate bronchodilating effect in asthma patients. J Chin Med Assoc 70: 265-268.

20. Zhang WP (2007) Effects of acupuncture on the pulmonary function and heart rate variability in different state of bronchial asthma. Zhen Ci Yan Jiu 32: 42-48.

21. Han J (2012) Observation on effect of acupuncture at Yuji (LU 10) on the pulmonary function of patients with bronchial asthma and immediate efficacy of relieving asthma. Zhongguo Zhen Jiu 32: 891-894.

22. Yang YQ, Chen HP, Wang Y, Yin LM, Xu YD, et al. (2013) Considerations for use of acupuncture as supplemental therapy for patients with allergic asthma. Clin Rev Allergy Immunol 44: 254-261.

23. Joos S, Schott C, Zou H, Daniel V, Martin E, et al. (2000) Immunomodulatory effects of acupuncture in the treatment of allergic asthma: a randomized controlled study. $J$ Altern Complement Med 6: 519-525.

24. Ukena D, Fishman L, Niebling WB (2008) Bronchial Asthma: Diagnosis and LongTerm Treatment in Adults. Dtsch Arztebl Int 105: 385-394.

25. Tan C, Zhang C, Gao D, Bai P, Wang J, et al. (2012) Impacts on the life quality of patients with bronchial asthma treated with acupuncture in terms of the lung and large intestine theory. Zhongguo Zhen Jiu 32: 673-677.

26. Reinhold T, Brinkhaus B, Willich SN, Witt C (2014) Acupuncture in patients suffering from allergic asthma: is it worth additional costs? J Altern Complement Med 20: 169177.

27. Feng JT, Hu CP, Li XZ (2007) Dorsal root ganglion: the target of acupuncture in the treatment of asthma. Adv Ther 24: 598-602.

28. Martin J, Donaldson AN, Villarroel R, Parmar MK, Ernst E, et al. (2002) Efficacy of acupuncture in asthma: systematic review and meta-analysis of published data from 11 randomised controlled trials. Eur Respir J 20: 846-852.

29. Medici TC, Grebski E, Wu J, Hinz G, Wütrich B, et al. (2002) Acupuncture and bronchial asthma: a long-term randomized study of the effects of real versus sham acupuncture compared to controls in patients with bronchial asthma. J Altern Complement Med 8: 737-750.

30. McCarney RW, Brinkhaus B, Lasserson TJ, Linde K (2004) Acupuncture for chronic asthma. Cochrane Database Syst Rev 1: CD000008.

31. McCarney RW, Lasserson TJ, Linde K, Brinkhaus B (2004) An overview of two Cochrane systematic reviews of complementary treatments for chronic asthma: acupuncture and homeopathy. Respir Med 98: 687-96.

Copyright: $@ 2018$ Zogopoulos P. This is an open-access article distributed under the terms of the Creative Commons Attribution License, which permits unrestricted use, distribution, and reproduction in any medium, provided the original author and source are credited. 